\title{
Class-specific depletion of lipid ion signals in tissues upon formalin fixation
}

Citation for published version (APA):

Vos, D. R. N., Bowman, A. P., Heeren, R. M. A., Balluff, B., \& Ellis, S. R. (2019). Class-specific depletion of lipid ion signals in tissues upon formalin fixation. International Journal of Mass Spectrometry, 446, 1-9. [116212]. https://doi.org/10.1016/j.ijms.2019.116212

Document status and date:

Published: 01/12/2019

DOI:

10.1016/j.ijms.2019.116212

Document Version:

Publisher's PDF, also known as Version of record

Document license:

Taverne

Please check the document version of this publication:

- A submitted manuscript is the version of the article upon submission and before peer-review. There can be important differences between the submitted version and the official published version of record.

People interested in the research are advised to contact the author for the final version of the publication, or visit the DOI to the publisher's website.

- The final author version and the galley proof are versions of the publication after peer review.

- The final published version features the final layout of the paper including the volume, issue and page numbers.

Link to publication

\footnotetext{
General rights rights.

- You may freely distribute the URL identifying the publication in the public portal. please follow below link for the End User Agreement:

www.umlib.nl/taverne-license

Take down policy

If you believe that this document breaches copyright please contact us at:

repository@maastrichtuniversity.nl

providing details and we will investigate your claim.
}

Copyright and moral rights for the publications made accessible in the public portal are retained by the authors and/or other copyright owners and it is a condition of accessing publications that users recognise and abide by the legal requirements associated with these

- Users may download and print one copy of any publication from the public portal for the purpose of private study or research.

- You may not further distribute the material or use it for any profit-making activity or commercial gain

If the publication is distributed under the terms of Article $25 \mathrm{fa}$ of the Dutch Copyright Act, indicated by the "Taverne" license above, 


\title{
Class-specific depletion of lipid ion signals in tissues upon formalin fixation
}

\author{
D.R.N. Vos, A.P. Bowman, R.M.A. Heeren, B. Balluff, S.R. Ellis* \\ The Maastricht Multimodal Molecular Imaging Institute (M4I), Maastricht University, 6229, ER, Maastricht, the Netherlands
}

\section{A R T I C L E I N F O}

\section{Article history:}

Received 23 April 2019

Received in revised form 23 July 2019

Accepted 30 August 2019

Available online 30 August 2019

\begin{abstract}
A B S T R A C T
Formalin fixation (FF) is widely used in pathology labs due to its ability to prevent tissue degradation and better preserve tissue morphology, thereby enabling long-term storage especially when combined with paraffin embedding. The detection of lipids and metabolites has recently been demonstrated from formalin-fixed paraffin-embedded (FFPE) tissues with matrix-assisted laser desorption/ionization mass spectrometry imaging (MALDI-MSI). However, the resulting lipid profiles differ markedly from those typically generated from fresh frozen tissue. To shed light onto the effects of formalin-induced modifications on lipids in tissues $24 \mathrm{~h}$-formalin-fixed and untreated mouse and rat kidney tissues were compared using time-of-flight matrix-assisted laser/desorption-mass spectrometry imaging (MALDIMSI) and Fourier transform MALDI-MSI combined with tandem mass spectrometry. These analyses revealed distinct and class-specific depletion of ion signals of certain lipid species upon formalin fixation, in particular for those containing amine functionalities. As MSI moves closer towards clinical application and necessitates increased analysis of formalin fixed tissues our results provide a route to rationalize the observed tissue-dependent lipid compositions observed from such tissues.
\end{abstract}

() 2019 Elsevier B.V. All rights reserved.

\section{Introduction}

Formalin fixation (FF), especially when combined with paraffin embedding, is the gold-standard approach for tissue preservation in pathology. The mechanism of preservation arises from the formalin-induced chemical modification of biomolecules, primarily via the reaction of formaldehyde with primary amines and thiols [1]. The most common outcome of this reaction is the crosslinking of biomolecules through the formation of methylene bridges which deactivates enzymatic activity enabling long term storage. Despite the induced chemical modifications a variety of molecular analysis methods have demonstrated that FF tissues can still yield rich biochemical information [2-7].

The detection of lipids and metabolites has recently been demonstrated from formalin-fixed paraffin-embedded (FFPE) tissues using matrix-assisted laser desorption/ionization mass spectrometry imaging (MALDI-MSI) [8,9]. Importantly, the resulting spectral profiles were found to enable tissue-type differentiation between two renal tumor types and revealed tissue-specific

\footnotetext{
* Corresponding author.

E-mail address: s.ellis@maastrichtuniversity.nl (S.R. Ellis).
}

distributions. However, the observed lipid profiles differed markedly from those typically generated from fresh frozen tissue, consisting of notably less detected lipid species than typically observed from fresh frozen tissue. These differences were largely attributed to lipid removal during the tissue processing steps that require organic solvents, as has also been reported using ultrahigh performance liquid chromatography [7]. However, it is reasonable to expect lipid alterations also originating from the fixation process itself [10]. Using extracts from cell cultures Cacciatore et al. reported that the majority of lipid species can still be detected after FF [7]. However, little information on the changing ion abundances and the possibility of class-specific depletion induced by FF was provided. Such depletion has previously been observed for FF brain extracts analyzed with LC-MS/MS [10]. Furthermore, previous MSI studies have observed spectral changes when analyzing FF tissues compared to fresh frozen tissues [11-13], although broad identification based on both high mass accuracy and MS/MS as well as detailed insight into the changing ion abundances of many lipid classes was not provided. Given the increasing role of MSI in clinical research $[14,15]$ and its potential for tissue classification based on local lipid composition it is important to understand the alterations in lipid profiles induced by FF and FFPE in tissues analyzed by MSI.

In this study we explicitly investigate the effects of FF on the 
nature and abundance of lipid signals observed from mouse and rat kidney tissue using time-of-flight MALDI-MSI and Fourier transform MALDI-MSI combined with tandem mass spectrometry. By exploiting the high mass accuracy and monoisotopic precursor ion selection these results unequivocally demonstrate the broad lipid class-specific depletion of ion signals observed during direct MSI analysis of untreated and FF tissues using high confidence, ontissue lipid identifcation. They also confirm previous studies demonstrating high quality MSI can be obtained for many lipid species from FF tissues. These results can help researchers rationalize and understand the observed MSI data from FF and FFPE tissues and determine if and how reflective they are of the native tissue environment.

\section{Experimental}

\subsection{Materials}

ULC/MS-CC/SFC grade methanol absolute and water was purchased from Biosolve Chimie SARL (Dieuze, France). Anhydrous chloroform, crystalline norharmane $\left(\mathrm{C}_{11} \mathrm{H}_{8} \mathrm{~N}_{2}\right)$, and zinc sulfate heptahydrate ( $99 \%$ A.C.S. reagent) was purchased from SigmaAldrich (Zwijndrecht, The Netherlands). Formalin fixative (3.7$4.0 \%$ ) containing zinc sulfate ( $\leq 1 \%$ according to manufacturer) and acetate buffered at $\mathrm{pH}$ 5.6-5.8 (Unifix) was purchased from Klinipath BV (Duiven, The Netherlands). Indium tin oxide-coated conductive (ITO) glass slides ( $\mathrm{R}_{S} 4-8 \Omega / \mathrm{sq}$ ) were purchased from Delta Technologies Ltd (Loveland, Colorado, USA). Hematoxylin solution modified according to Gill and entellan ${ }^{\circledR}$ new was purchased from Merck KGaA (Darmstadt, Germany). Eosine-Y, Alcholic was purchased from Avantor ${ }^{\circledR}$ Performance Materials B.V. (Deventer, the Netherlands), and cover slips purchased from Thermo Scientific (Waltham, Massachusetts, USA).

\subsection{Sample preparation}

Two mouse kidneys were sourced from the same animal at John Hopkins University School of Medicine under the ethical approval by the Institutional Animal Care and Use Committee at the Johns Hopkins University School of Medicine (MO17M190). Two rat kidneys were sourced from the same rat at Maastricht University Medical Centre under the ethical approval by the institution's review board, the Animal Ethical committee, the Inspection for Animal Welfare and the Dutch Central Committee of Animal experiments (AVD107002016720). All organs were snap frozen by immediately transferring them into liquid nitrogen after removal and stored at $-80^{\circ} \mathrm{C}$ until fixation and/or sectioning. One kidney of each animal was fixed for $24 \mathrm{~h}$ in a zinc sulfate buffered formalin fixation solution following a standard pathology protocol. Prior to fixation tissue was first acclimated for an hour at $-20^{\circ} \mathrm{C}$ after removing it from $-80^{\circ} \mathrm{C}$. This was followed by moving the sections to room temperature for an hour to ensure they were completely thawed before emerging them in the fixation solution. After fixation for $24 \mathrm{~h}$ the fixation solution was removed and the fixed kidneys were stored back at $-80^{\circ} \mathrm{C}$ until sectioning. The other kidney of each animal was left untreated and kept at $-80^{\circ} \mathrm{C}$ until sectioning. Both fixed and untreated kidneys were transversally sectioned at the thickest part of the kidneys at $-20^{\circ} \mathrm{C}$ on a Microm HM525 cryotome (Thermo Fisher Scientific, Waltham, Massachusetts, USA). Sections were cut $10-12 \mu \mathrm{m}$ thick and thaw-mounted onto ITO glass slides.

For the MALDI-TOF measurements slides were coated with 8 layers of norharmane matrix solution $(7 \mathrm{mg} / \mathrm{mL}$ norharmane in $2: 1$ chloroform:methanol (v:v)). An automated TM-Sprayer (HTX
Technologies, LLC, North Carolina, USA) was used for matrix application with a flowrate of $0.12 \mathrm{~mL} / \mathrm{min}, 30 \mathrm{~s}$ drying time between each layer and a temperature of $30^{\circ} \mathrm{C}$. Slides for MALDIOrbitrap analysis were prepared using identical parameters, but coated with 15 layers of norharmane matrix.

After MSI slides were subsequently washed with 2 x 70\% ethanol followed by Milli Q water for 3 min each before staining with hematoxylin for $3 \mathrm{~min}$. Excess of hematoxylin was removed with a 3 min water wash followed by eosin staining for $30 \mathrm{~s}$ followed by another 3 min water wash to remove the excess of eosin. This was followed by a $1 \mathrm{~min}$ ethanol wash and $30 \mathrm{~s}$ xylene wash before coverslips were placed on top of the slides using Entellan as a mounting medium to adhere the coverslips to the sections. Optical images of the H\&E stained tissues were acquired using a MIRAX scanner (Zeiss, Breda, The Netherlands).

Washing of the untreated rat kidneys with zinc sulfate was performed by submerging a slide with rat kidney sections in a $1 \%$ zinc sulfate solution in water for $30 \mathrm{~s}$.

\subsection{MALDI-MSI measurements}

All MALDI-TOF-MSI was acquired at $50 \mu \mathrm{m}$ spatial resolution on a rapifleX MALDI-ToF/ToF instrument [16] (Bruker Daltonik GmbH, Bremen, Germany). Tissues from each kidney (mouse and rat) were analyzed in both negative-ion and positive-ion mode using adjacent sections for each polarity and data acquired in the $m / z$ range of 200-2000. The laser scanned across a $45 \times 45 \mu \mathrm{m}^{2}$ area using a $50 \times 50 \mu \mathrm{m}^{2}$ raster size with 200 laser shots summed for each position. Laser power and focus were optimized for each tissue section.

MSI experiments at high mass resolution and accuracy were performed using an Orbitrap Elite Mass Spectrometer (Thermo Fisher, Bremen, Germany) equipped with an ion-funnel based MALDI interface (Spectroglyph LLC, Kennewick, WA, USA) as recently reported [17]. Three untreated and FF mouse kidney sections were each measured in the negative-ion mode at a pixel size of $50 \times 50 \mu \mathrm{m}^{2}$ between $\mathrm{m} / z$ 200-2000. Data was acquired using a nominal mass resolution of 240,000 (FWHM at $m / z 400$ ), an injection time of $250 \mathrm{~m} \mathrm{~s}$ and a laser repetition rate of $1 \mathrm{KHz}$. In addition, one FF and unfixed section from both mouse and rat kidney tissues was analyzed in positive- and negative-ion mode using the data-dependent acquisition (DDA)-imaging method as recently described [18]. Data was acquired using a stage step size of $25 \times 50 \mu \mathrm{m}^{2}$. This approach acquires one full-scan Fourier transform mass spectrometry spectrum in the Orbitrap and one DDA-MS/MS scan in the ion trap at each $50 \times 50 \mu \mathrm{m}^{2}$ area of tissue. Acquisition in this manner allows simultaneous acquisition of both a high mass resolving power MSI dataset, accurate mass measurements of all detected lipids and ion trap MS/MS spectra of lipids using monoisotopic precursor selection. DDA-imaging was performed in both negative-ion and positive-ion mode on consecutive tissue sections and data acquired within the $m / z$ range $200-2000$ using an ion injection time of $250 \mathrm{~m} \mathrm{~s}$ and a nominal mass resolution of 240,000 (FWHM at $m / z 400$ ) for the Orbitrap (MSI) scans. Typical mass accuracy was $\leq 2 \mathrm{ppm}$. Both ion activation and mass analysis of the fragment ions were performed in the dual pressure linear ion trap using resonant collision-induced dissociation (CID). In negative-ion mode fragmentation was performed using a normalized collision energy of 38 (manufacturer units) and an activation q of 0.25 . In positive-ion mode fragmentation was performed at a normalized collision energy of 30 (manufacturer units) and an activation q of 0.17 to allow for the detection of the low $\mathrm{m} / \mathrm{z}$ fragment ion at $\mathrm{m} / \mathrm{z}$ 184 produced from PC and SM lipids. 


\subsection{MSI data analysis}

MALDI-ToF data was imported into SCiLS software version 2016b (SCiLS GmbH, Bremen, Germany) for further analysis. To define regions-of-interest (ROIs) and extract region-specific spectra MSI data was co-registered with the optical images of the post-MSI H\&E-stained tissues sections.

High mass resolution imaging data (excluding DDA-imaging data) was converted into imzML using ImageInsight software (Spectroglyph LLC, Kennewick, WA, USA) and imported into SCiLS software and the publicly accessible METASPACE annotation platform [19]. Regions-of-interest (ROI) were manually defined by visual correlation with the $\mathrm{H} \& \mathrm{E}$ stained tissues. ROI-spectra were then extracted from the cortex and medulla regions of each section and loaded in to mMass software [20] for peak picking and peak intensity determination. For MSI data visualisation this data was also converted to mzXML format using RawConverter software [21] and visualized using in-house scripts for MATLAB (version R2014a, The MathWorks, Natick, USA) as previously described [17].

DDA-imaging data was converted to mzXML format using a RawConverter [21] and analyzed using in-house scripts for MATLAB (version R2014a, The MathWorks, Natick, USA) as described above and previously [18]. DDA-MS/MS data was analyzed using Thermo Xcalibur 2.3.26 (Thermo Fisher, Bremen, Germany).

\subsection{Lipid identification}

Accurate $m / z$ values for precursor ions obtained from the Orbitrap data were used to first determine the sum-composition formula of the detected lipid species. Supporting sum-composition fragments or those allowing assignment of individual acyl chains were acquired from theDDA-MS/MS data of the untreated tissues. Assignment of the fragments in the MS/MS spectra was performed using the online ALEX $^{123}$ database (http://alex123.info/ALEX123/ MS.php) and the proposed nomenclature by Pauling et al. for all identified fragments reported in the ALEX ${ }^{123}$ database [22]. In some cases several supporting fragments not reported in the ALEX ${ }^{123}$ databases were also observed and annotated based on prior literature reports [23].

Selected MS/MS measurements were performed using higherenergy collisional dissociation (HCD) combined with Orbitrap detection of fragment ions to aid in the identification of several unknown ion species. HCD spectra were obtained using a $1.0 \mathrm{Da}$ isolation window, a normalized collision energy of 40 (manufacturer units) and detection of fragment ions at a mass resolution setting of 240,000 (FWHM at $m / z$ 400) in the Orbitrap. HCD-MS/MS spectra were acquired from a selected list of precursor ions using a $5000 \mathrm{~ms}$ injection time while continuously moving the stage over the tissue at $1 \mathrm{~mm} / \mathrm{s}$. Data for each precursor was acquired for $2 \mathrm{~min}$ prior to spectral averaging for analysis.

For both broader identification of sum-composition lipid species and determining the percentage contribution of each lipid class to the total identified lipid signal in the mouse kidney dataset the ALEX $^{123}$ software package was used $[18,24,25]$. For each replicate an average spectrum of each sample was first generated. Average spectra were then used to identify lipids using an $m / z$ tolerance of $\pm 2.5 \mathrm{mDa}$, a relative intensity threshold of $0.1 \%$ base peak intensity (defined as the most intense identified lipid in the averaged spectra) and searching for the following lipid classes as [M-H] $]^{-}$ions: PA, PE, PS, PI, PG, their corresponding ether and lyso variants, SHexCer, Cer, GM1, GM2, GM3 and CL. The percentage contribution of each lipid class to the total identified lipid signal was then calculated using Tableau Desktop version 2018.2 (Tableau Software, CA, USA). Finally the percentage contribution of each class within fixed and unfixed replicate was averaged to generate the final dataset.

\section{Results \& discussion}

Three transversal sections taken from both untreated and formalin fixed (FF) mouse kidney tissue were analyzed using MALDI-TOF-MSI in both negative-ion and positive-ion mode. Analysis of this data revealed drastic differences in the mean spectra generated from the cortex and medulla regions from untreated and FF tissues (Fig. 1). Spectra from individual sections are provided in Supporting Information Figs. S1 and S2. In particular, the depletion of a variety of lipid-related peaks is observed in the negative-ion mode upon FF, especially in the $m / z 700-850$ range. We note the additional freeze/thaw cycle undergone by the fixed tissue is expected to have a negligible influence on the observation made during this study. Previous work has demonstrated that the primary artefact induced by repetitive freeze/thaw cycles is an increase in free fatty acid signals, and this effect becomes more pronounced with more cycles. Given only one additional freeze/ thaw cycle was performed, and that no increase in free fatty acid signals is observed in the fixed tissue, this effect on the data analysis can be excluded.

Fig. 2 shows selected ion images and narrow $m / z$ ranges acquired from a single FF and untreated mouse kidney tissue sections in the negative-ion mode. These data reveal in greater the detail the specific depletion of certain lipid signals upon FF. The corresponding mean spectra and MSI data from all replicates are provided in Fig. S3 and are consistent with the data shown in Fig. 2. Fig. 2A-C shows example ion distribution images and spectral ranges that show little alteration upon FF, Importantly, and in line with earlier reports $[8,11,12]$, consistent ion images are observed from both fixed and unfixed tissues indicating that the solventbased fixation process does not significantly alter the spatial distributions of lipids detectable in FF tissues. In contrast, Fig. 2D and E shows example ion images and spectral ranges acquired in the negative-ion mode that are significantly depleted upon $\mathrm{FF}$ (e.g., $m / z$ 766.5 and 810.5). Several ions are also observed at higher abundance upon FF, for example $m / z 799.5$ shown in Fig. $2 \mathrm{~F}$, that is observed primarily from the cortex regions of the kidney. Analogous changes are also observed from identically-treated rat kidney tissues (Figs. S4-S5). The potential influence on the obtained spectra by the zinc sulfate present in the fixation solution was also investigated. Spectra acquired from fresh frozen tissue with and without washing in $1 \%$ aqueous zinc sulfate solution are provided in Supporting Fig. S6. Although a strong desalting effect was observed in the washed tissue leading to a reduction of $[\mathrm{M}+\mathrm{Na}]^{+}$and $[\mathrm{M}+\mathrm{K}]^{+}$ions, crucially, the detected lipid species and classes were very similar, providing evidence the observed spectral changes are not the result of the zinc sulfate within the fixation solution.

The above results provide evidence of class-specific lipid depletion of ion signals upon FF. To elucidate the specific lipid classes affected by FF we further analyzed both FF and fresh frozen mouse and rat kidney tissues using high mass resolution MALDIMSI and MS/MS. Fig. 3 shows the average ion intensities for a variety of lipid species detected in the cortex and medulla regions of three untreated and FF mouse kidney sections. MS/MS data supporting the molecular-lipid species lipid identifications are provided in the Supporting Information (Figs. S7-S12). As these comparisons are made from the same tissue regions comprising similar original compositions the observed differences must arise via FF-induced processes. Fig. $3 \mathrm{~A}$ and $\mathrm{B}$ shows the intensities of several phosphatidylethanolamines (PE) and phosphatidylserines (PS) lipid species. Both PE and PS lipids show a dramatic and statistically significant reduction in signal intensity after FF that is consistent with prior observations using both MSI and LC-MS/MS 


\section{A - Negative mode}

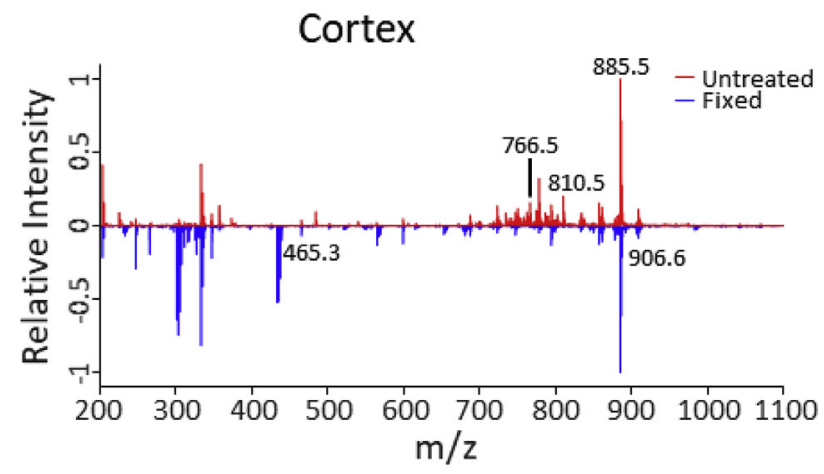

\section{B - Positive mode}

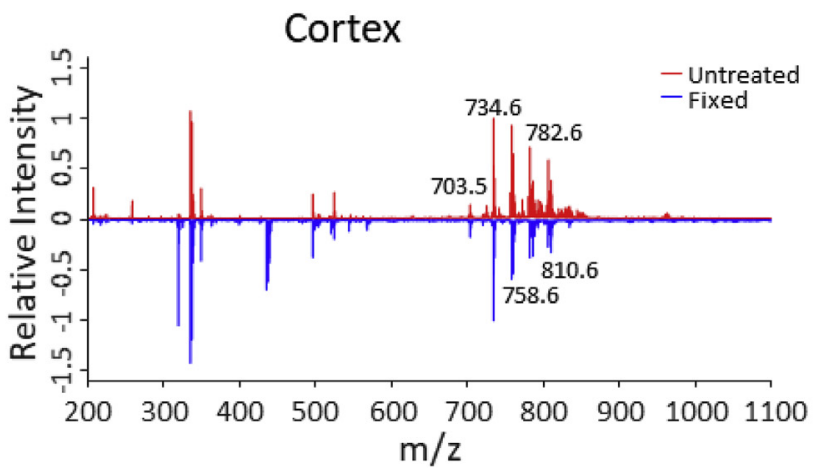

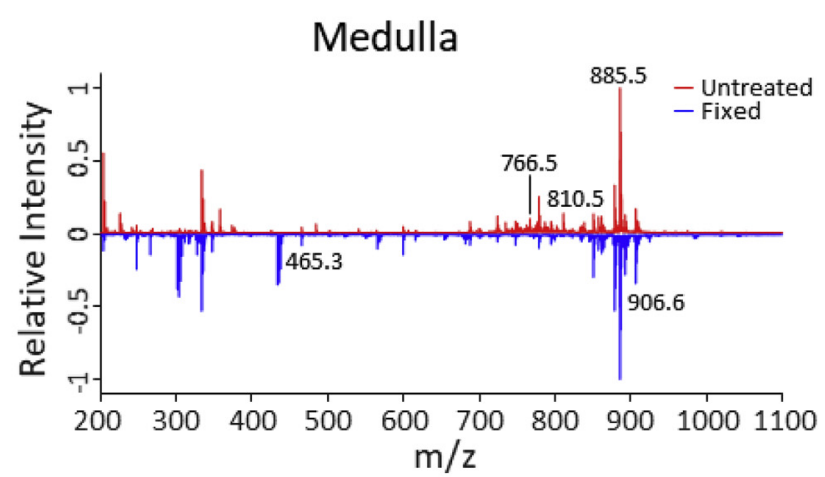

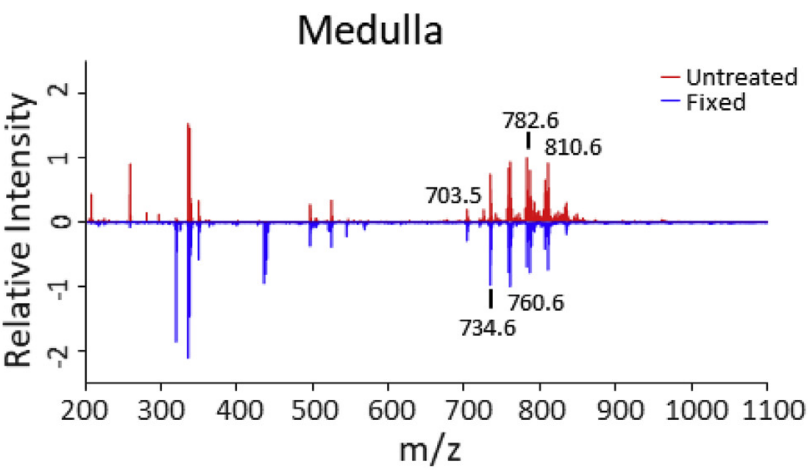

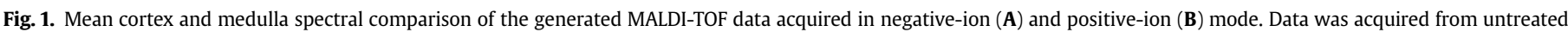

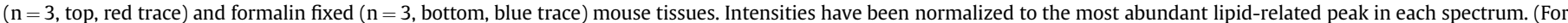
interpretation of the references to colour in this figure legend, the reader is referred to the Web version of this article.)

$[10,11]$ and the known mechanism of formalin fixation. More specifically, formalin reacts with the primary amine groups of the PE and PS headgroups, effectively crosslinking the lipids with other amine-containing lipids and proteins. This crosslinking is hypothesized to inhibit the extraction of free lipids into the matrix and their subsequent ionization by MALDI. Phosphatidic acid (PA) species show a varied response upon fixation (Fig. 3C). The ions corresponding to $\left[\mathrm{PA}\left(18: 0 \_18: 1\right)-\mathrm{H}\right]^{-}$and $\left[\mathrm{PA}\left(18: 0 \_22: 4\right)-\mathrm{H}\right]^{-}$ remaining relatively unaffected by fixation. This can be rationalized by the lack of an amine group in PA lipids. In contrast [PA(18:0_20:4)-H] $]^{-}$shows an apparent $\sim 2$ fold reduction upon FF. This result is initially surprising due to the lack of an amine group that can undergo crosslinking. However, PA lipids can also form via in-source fragmentation of other phospholipids, most significantly from PS lipids following loss of the serine headgroup (-87 Da). Thus part of the $\left[\mathrm{PA}\left(18: 0 \_20: 4\right)-\mathrm{H}\right]^{-}$ion population likely arises from the abundant [PS(18:0_20:4)-H] $]^{-}$species that is reduced upon FF (Fig. 3B). Thus results for PA lipids reflect a convolution of both endogenous PA lipids and those arising from in-source fragmentation of other lipid species. Phosphatidylinositol (PI) lipids showed a $\sim 2$-fold reduction after FF (Fig. 3D). We speculate this is due to either minor degradation of endogenous lipids during the fixation process and/or partial reaction of the hydroxyl groups of PI with formalin. Phosphatidylglycerol (PG) remains largely unaffected by fixation (Fig. 3E). This is in contrast to previous work that has reported both a decrease [10] and an increase [11] in PG ion intensities after FF, although we note we only observed one PG species at sufficient intensity for analysis. Interestingly both sulfatides (SHexCer, Fig. 3F) and cholesterol sulfate (CholS, Fig. 3G) exhibited higher intensities after FF within the medulla. This observation can possibly be explained by an increase in ionization efficiency, resulting from the decreased amounts of other phospholipids classes that may lead to charge competition and ionization suppression. A similar effect resulting in increased signals for glycosphingolipids following removal of phospholipids using a phospholipase $C$ digestion protocol has been reported previously by Vens-Cappell et al. [26].

From a broader lipidomics perspective, Fig. 4 shows the changes in relative contributions to the total identified sum-composition lipid signal ( $c f .$, "Lipid Identification" section in the Methods) obtained from both fixed and unfixed mouse kidney tissues in the negative-ion mode $\mathrm{MS}^{1}$ data (i.e., lipids are assigned based on accurate $m / z$ values). The full list of identified sum-composition lipid species is provided in Supporting Information Table S1. Consistent with the above, Fig. 4 shows an increased contribution from sulfatides and a reduced contribution from a variety of lipid species, in particular PI, PE and PS species. We note that the relative decrease in PE species appears to be less than that shown in Fig. 3. This is due to the $\mathrm{MS}^{1}$-based identification routine incorrectly assigning several ions originating from the in-source fragmentation of PC species to their corresponding $\left[\mathrm{M}-\mathrm{CH}_{3}\right]^{-}$ions as isomeric deprotonated PE lipids. For example based only on accurate mass $\mathrm{m} / \mathrm{z}$ 718.5386 is assigned as $[\mathrm{PE}(34: 0)-\mathrm{H}]^{-}$. However, MS/MS data reveals an almost exclusive presence of 16:0-related fragments, suggesting this ion is instead $\left[\mathrm{PC}(32: 0)-\mathrm{CH}_{3}\right]^{-}$(data not shown). Fig. 5 shows the corresponding ion distribution images for all species discussed above in Fig. 3 and reveal consistent ion distributions for all lipid species observed in both untreated and FF 

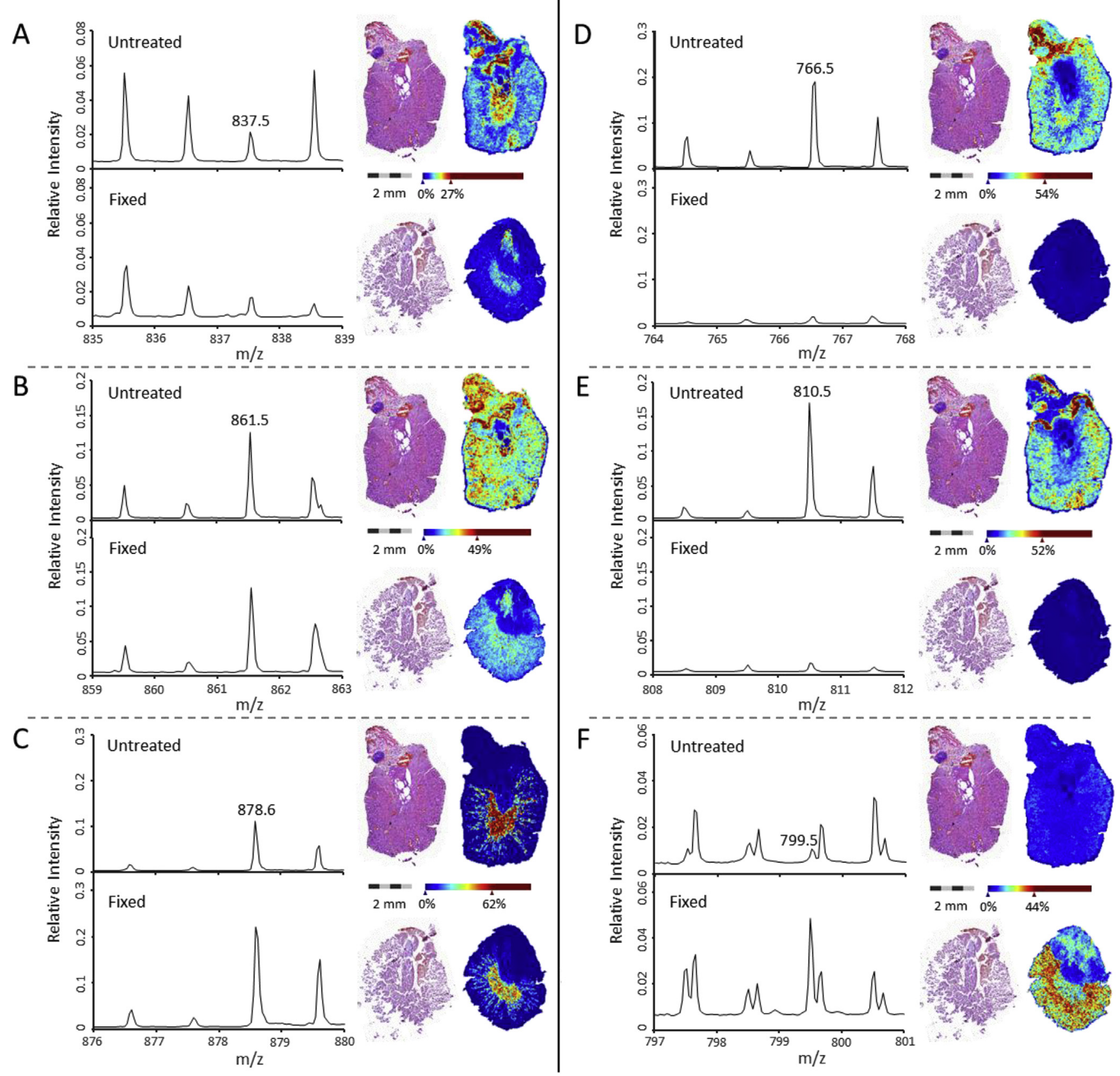

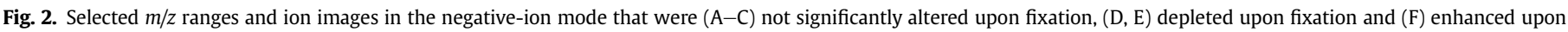

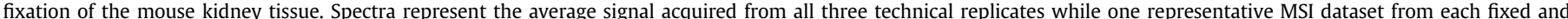

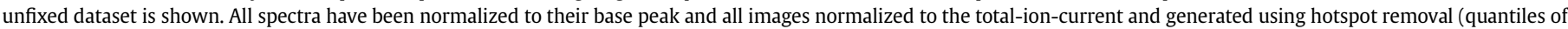

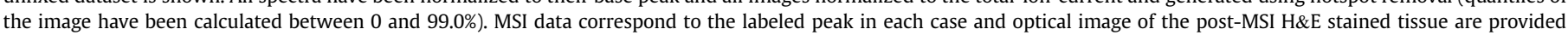

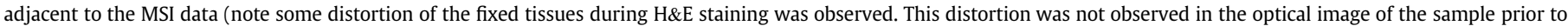

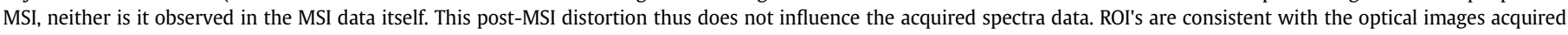
prior to MSI where cortex and medulla regions are also visible).

tissues. For interested readers the complete negative-ion mode datasets from each mouse kidney replicate can be accessed via the METASPACE online annotation platform (https://metaspace2020. eu/) [27]. Similar class-dependent depletion of lipid signals was also obtained in rat kidney tissue sections (Supporting Information Figs. S13 and S14).

An interesting observation in negative-ion mode was the presence of the $\left[\mathrm{M}+\mathrm{HSO}_{4}\right]^{-}$adducts for sphingomyelin (SM) in the fixed mouse and rat kidney tissue. From mouse kidney tissue, these ions were observed in the Orbitrap data at $\mathrm{m} / \mathrm{z} 799.5274$ and $\mathrm{m} / \mathrm{z}$
911.6524 ( $m / z 799.5$ is also shown in Figs. 1 and 2). The measured $\mathrm{m} / \mathrm{z}$ values provided in the high resolution Orbitrap analysis yielded no logical matches in either the LIPIDMAPS database or the ALEX ${ }^{123}$ lipid calculator. However, HCD-MS/MS spectra for these ions (Supporting Information Figs. S15-S18) revealed a sole fragment ion at $m / z 96.9603$ for both precursors which was assigned to the $\mathrm{HSO}_{4}^{-}$anion (mass errors $\leq 2.5 \mathrm{ppm}$ ). This anion formed following neutral losses of 702.5662 and 814.6909 from the precursor ions at $\mathrm{m} / \mathrm{z} 799.5274$ and 911.6524 , respectively. These neutral losses correspond to the neutral masses of the sphingomyelin (SM) 
A - PE

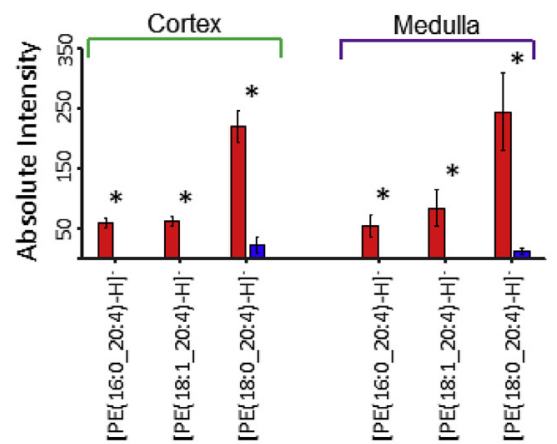

$\mathrm{D}-\mathrm{PI}$

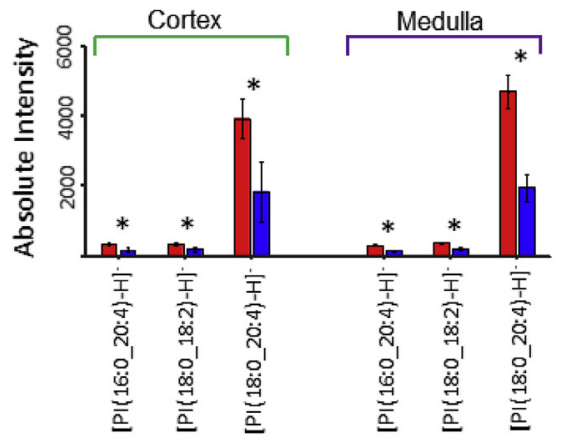

B - PS

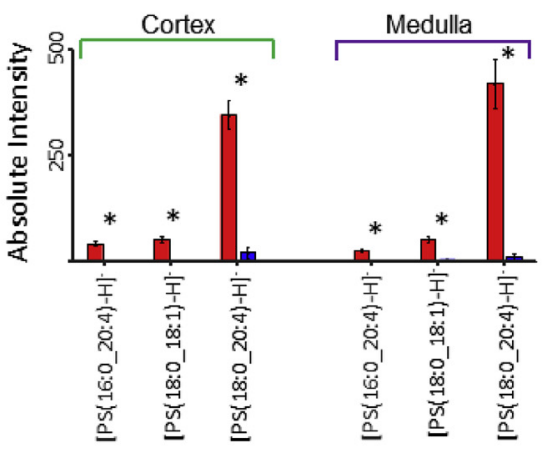

$E-P G$

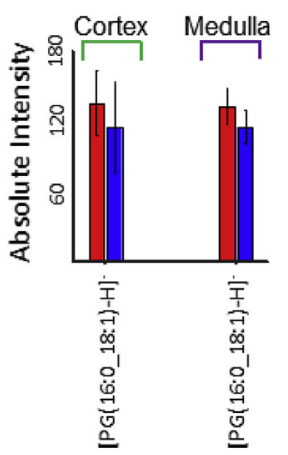

F - SHexCer
C - PA

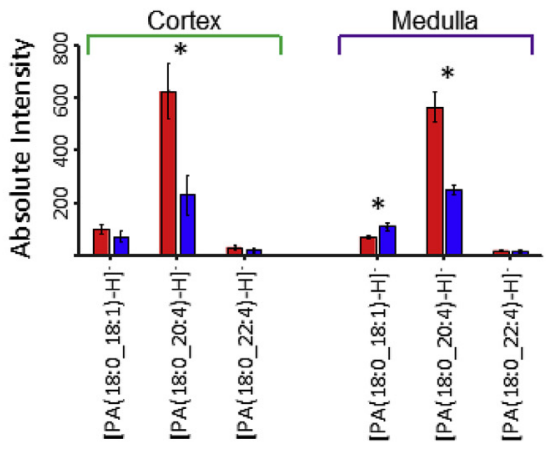

G - Chols

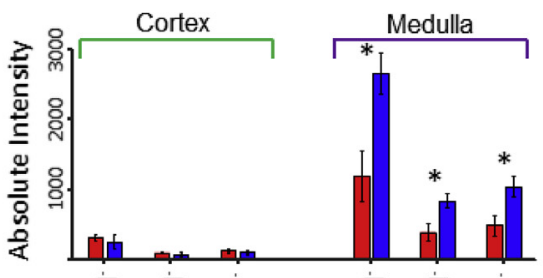

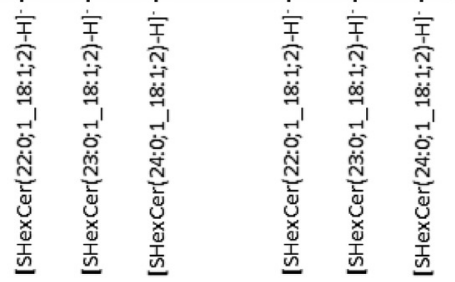

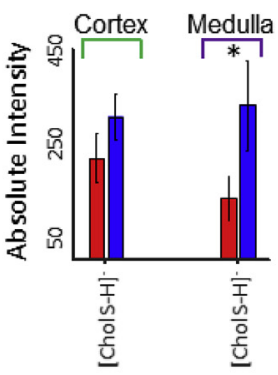

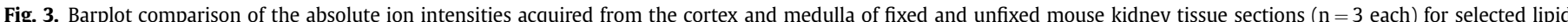

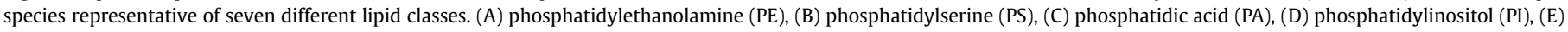

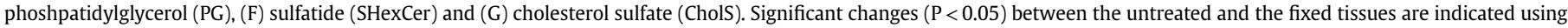
an asterix. Error bars represent \pm 1 standard deviation.

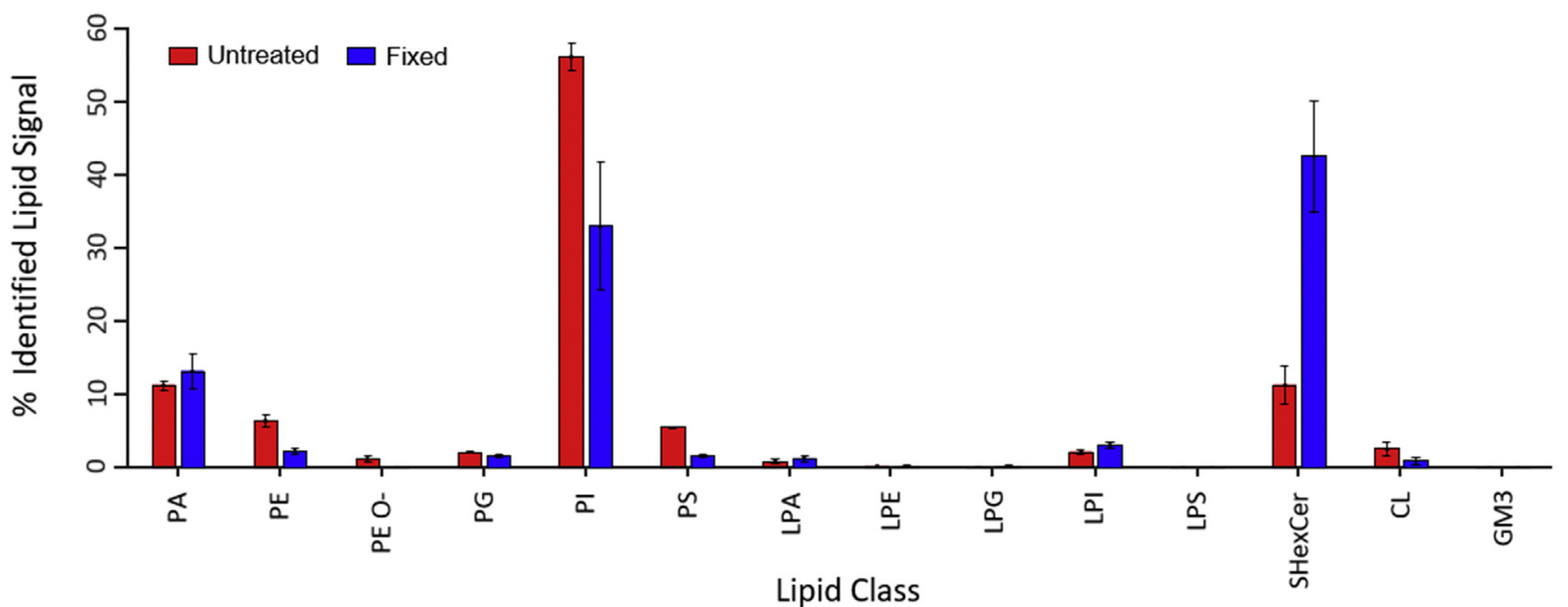

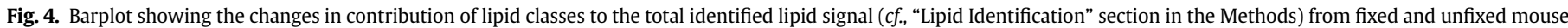
kidney tissue $(n=3$ each). Error bars represent \pm 1 standard deviation.

species SM 34:1;2 and SM 42:1;2 (-2.8 ppm and -3.0 ppm), respectively, providing strong evidence for the assignment of these ions as $\left[\mathrm{M}+\mathrm{HSO}_{4}\right]^{-}$ions of these SM lipids. The source of the hydrogen sulfate is likely from the zinc sulfate containing formalin solution. The ability to detect SM lipids in the negative-ion mode, in addition to the more acidic sphingolipids such as SHexCers, can allow the direct correlation of their distributions within a single dataset that could serendipitously prove beneficial in the study of 


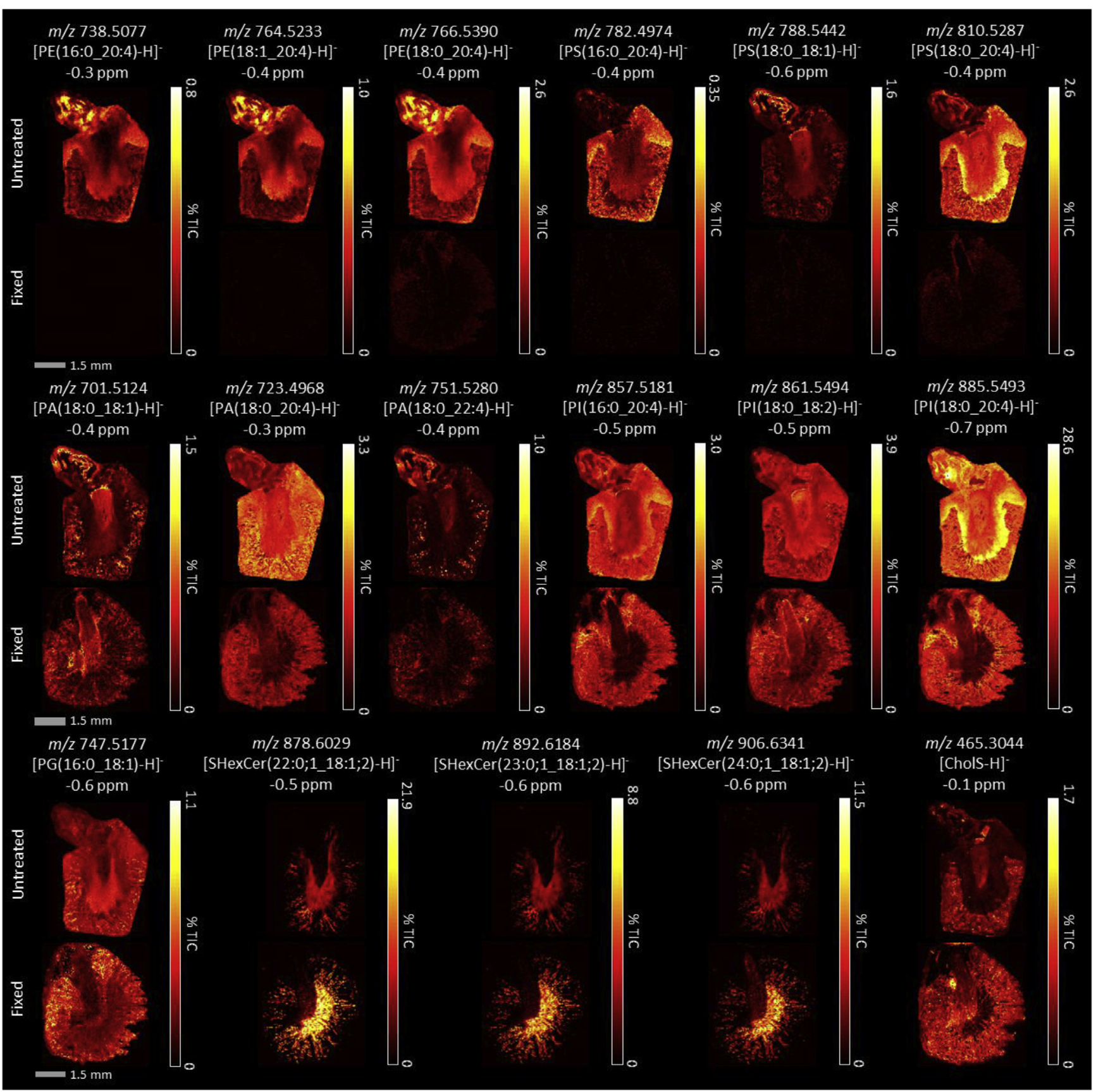

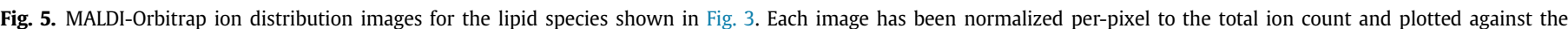

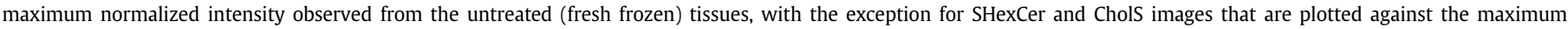
normalized intensity observed from the FF tissues. Confirmatory MS/MS spectra are provided in the Supporting Information.

sphingolipid metabolism.

Taken together, the above results rationalize the observations in the initial MALDI-TOF data shown in Figs. 1 and 2. In Fig. 2 the presence of ions at $m / z 837.5,861.5$ and $m / z 878.5$ that are not massively depleted upon FF is observed. Using the DDA-imaging data these ions are identified as $\left[\mathrm{PI}\left(16: 0 \_18: 1\right)-\mathrm{H}\right]^{-},\left[\mathrm{PI}\left(18: 0 \_18: 2-\right.\right.$ $\mathrm{H}]^{-}$and [SHexCer(22:0; 1_18:1;2)] $]^{-}$, respectively and are lipid classes that do not contain an amine group and are thus expected to be tolerant to FF. On the other hand, the ions observed at $\mathrm{m} / \mathrm{z} 766.5$ and $m / z 810.5$ (Fig. 2D and E) are identified as [PE(18:0_20:4)-H] and [PS(18:0_20:4)-H] $]^{-}$ions, respectively. These both correspond to amine-contacting lipids and their signal depletion is consistent with the known mechanisms of fixation. Finally, as described above, the ion detected at $m / z 799.5$ in Fig. 2 is assigned as a $\left[\mathrm{M}+\mathrm{HSO}^{-}\right.$ion of SM $34: 1 ; 2$ and is an artefact of the sulfatecontaining formalin solution.

In the positive-ion mode the combined high mass accuracy and MS/MS measurement enabled the differences shown in Fig. 1B to be identified. As expected positive-ion mode spectra were dominated by PC and SM lipids in both FF and untreated tissues. Although at first look the spectra appear similar, being dominated by abundant $[\mathrm{M}+\mathrm{H}]^{+}$ion in both cases, an increase in alkali adducts, most notably $[\mathrm{M}+\mathrm{Na}]^{+}$ions, is also observed in the untreated tissues. The shift towards protonated ions in the fixed mouse and rat tissue is 
attributed to the aqueous fixation solution which effectively removes endogenous sodium and potassium from the tissue. This effect is analogous to studies utilizing aqueous washing to desalt tissues prior to MSI $[28,29]$ and is consistent with work demonstrating a shift in adduct formation of PC lipids before and after fixation, with the specific shift depending on the composition of the formalin fixation solution $[11,12]$. However, $[\mathrm{M}+\mathrm{Na}]^{+}$and $[\mathrm{M}+\mathrm{H}]^{+}$ adducts of different PC lipid species can remain unresolved even with the high mass resolving power realized here. To demonstrate the shift towards protonated species in the FF tissue we have analyzed the CID-MS/MS spectra acquired from precursor ions of $m / z 782.57$ and $m / z 810.60$ in both positive-ion mode datasets. These two ion populations represent isobaric combinations of [PC $36: 4+\mathrm{H}]^{+} /[\mathrm{PC} 34: 1+\mathrm{Na}]^{+}$and $[\mathrm{PC} 38: 4+\mathrm{H}]^{+} /[\mathrm{PC} 36: 1+\mathrm{Na}]^{+}$ respectively. In the untreated tissue both the 59 and 183 neutral loss fragments are observed at high abundance and are attributed to the losses of $\mathrm{N}\left(\mathrm{CH}_{3}\right)_{3}(-59 \mathrm{Da})$ and phosphocholine $(-183 \mathrm{Da})$ respectively (Supporting Information Figs. S19a and S20a (from mouse tissue) and S21a, S22a (from rat tissue)). These fragment ions are known to form to a far greater extent for alkali adducted PC lipids compared to their protonated variants [30], thereby providing evidence for a significant population of $[\mathrm{M}+\mathrm{Na}]^{+}$ions. By contrast, in the fixed tissue the $\mathrm{m} / \mathrm{z} 184$ fragment is formed almost exclusively and is the main expected fragment from protonated PC lipids (Supporting Information Figs. S19b and S20b (from mouse tissue) and S21b, S22b (from rat tissue). These data demonstrate that FF does not significantly influence the detection of PC and SM lipids in positive-ion mode but simply results in a shift in adduct profile. In this case this results in the almost exclusive formation of protonated PC lipids in the fixed tissue.

\section{Conclusion}

Using MALDI-MSI combined with accurate mass measurements and MS/MS we have systematically investigated the effect of formalin fixation on lipids in kidney tissue, a widely used model tissue for MSI studies. In line with the known crosslinking mechanism of formalin our results show a significant depletion of ion signals from primary amine containing phospholipids, namely PE and PS that is consistent with earlier reports [10,11]. We did not observe potential intermediate reaction products of the fixation process such as $\mathrm{N}$-formylation $\mathrm{N}$-methylation as previously reported for LC-MS [10] or cross-linked lipid species. The latter observation can be rationalized by the generation of a cross-linked network of lipids and proteins. Although the depleted lipids only contain a single amine group they may still form an interconnected covalent network with other cross-linked species that is not efficiently extracted into the matrix, ionized, and that exceeds the accessible mass range. It is noteworthy that despite the solventbased fixation procedure high quality MSI data can still be acquired from fixed tissues for many different classes with minimal delocalisation compared to unfixed tissues (e.g., Figs. 2 and 4). The potential recovery of cross-linked lipids by optimized antigen retrieval steps may provide an exciting future avenue of research to increase the lipid coverage achievable when analyzing FF tissues.

FF is not used in isolation, but routinely in combination with paraffin embedding (FFPE tissues). Given the recent demonstration of lipid detection from clinically-relevant FFPE tissues using MALDI-MSI and the potential diagnostic power of such analyses [8] our results can help rationalize the detected lipid species in these applications (independent of potential losses arising from the paraffin embedding process itself) and provide insight into the biochemical conclusions that can, and cannot, be drawn in these studies.

\section{Acknowledgements}

This work has been made possible with the financial support of the LINK program of the Dutch province of Limburg and has been funded by ITEA and RVO by means of project numbers ITEA151003/ ITEA 14001. A.P.B, R.M.A.H and S.R.E acknowledge financial support from Interreg $V$ EMR and the Netherlands Ministry of Economic Affairs within the "EURLIPIDS" project (project number EMR23). We are grateful to the Glunde lab in the Department of Radiology at Johns Hopkins, Baltimore, for providing mouse kidney tissue.

\section{Appendix A. Supplementary data}

Supplementary data to this article can be found online at https://doi.org/10.1016/j.ijms.2019.116212.

\section{References}

[1] R. Thavarajah, V.K. Mudimbaimannar, J. Elizabeth, U.K. Rao, K. Ranganathan, Chemical and physical basics of routine formaldehyde fixation, J. Oral Maxillofac. Pathol. 16 (2012) 400-405.

[2] C.M. Krishna, G.D. Sockalingum, R.A. Bhat, L. Venteo, P. Kushtagi, M. Pluot, M. Manfait, FTIR and Raman microspectroscopy of normal, benign, and malignant formalin-fixed ovarian tissues, Anal. Bioanal. Chem. 387 (2007) 1649-1656.

[3] C.M. Krishna, G.D. Sockalingum, B.M. Vadhiraja, K. Maheedhar, A.C. Rao, L. Rao L. Venteo, M. Pluot, D.J. Fernandes, M.S. Vidyasagar, V.B. Kartha, M. Manfait, Vibrational spectroscopy studies of formalin-fixed cervix tissues, Biopolymers 85 (2007) 214-221.

[4] A.D. Kelly, S.B. Breitkopf, M. Yuan, J. Goldsmith, D. Spentzos, J.M. Asara, Metabolomic profiling from formalin-fixed, paraffin-embedded tumor tissue using targeted LC/MS/MS: application in sarcoma, PLoS One 6 (2011) e25357.

[5] C. Steiner, J.C. Tille, J. Lamerz, S. Kux van Geijtenbeek, T.A. McKee, M. Venturi, L. Rubbia-Brandt, D. Hochstrasser, P. Cutler, P. Lescuyer, A. Ducret, Quantification of HER2 by targeted mass spectrometry in formalin-fixed paraffinembedded (FFPE) breast cancer tissues, Mol. Cell. Proteom. 14 (2015) 2786-2799.

[6] A. Wojakowska, M. Chekan, L. Marczak, K. Polanski, D. Lange, M. Pietrowska, P. Widlak, Detection of metabolites discriminating subtypes of thyroid cancer: molecular profiling of FFPE samples using the GC/MS approach, Mol. Cell. Endocrinol. 417 (2015) 149-157.

[7] S. Cacciatore, G. Zadra, C. Bango, K.L. Penney, S. Tyekucheva, O. Yanes, M. Loda, Metabolic profiling in formalin-fixed and paraffin-embedded prostate cancer tissues, Mol. Cancer Res. 15 (2017) 439-447.

[8] A. Buck, A. Ly, B. Balluff, N. Sun, K. Gorzolka, A. Feuchtinger, K.P. Janssen, P.J. Kuppen, C.J. van de Velde, G. Weirich, F. Erlmeier, R. Langer, M. Aubele, H. Zitzelsberger, M. Aichler, A. Walch, High-resolution MALDI-FT-ICR MS imaging for the analysis of metabolites from formalin-fixed, paraffinembedded clinical tissue samples, J. Pathol. 237 (2015) 123-132.

[9] A. Ly, A. Buck, B. Balluff, N. Sun, K. Gorzolka, A. Feuchtinger, K.P. Janssen, P.J. Kuppen, C.J. van de Velde, G. Weirich, F. Erlmeier, R. Langer, M. Aubele, H. Zitzelsberger, L. McDonnell, M. Aichler, A. Walch, High-mass-resolution MALDI mass spectrometry imaging of metabolites from formalin-fixed paraffin-embedded tissue, Nat. Protoc. 11 (2016) 1428-1443.

[10] M. Gaudin, M. Panchal, S. Ayciriex, E. Werner, A. Brunelle, D. Touboul, C. Boursier-Neyret, N. Auzeil, B. Walther, C. Duyckaerts, O. Laprevote, Ultra performance liquid chromatography - mass spectrometry studies of formalininduced alterations of human brain lipidome, J. Mass Spectrom. 49 (2014) 1035-1042.

[11] C.L. Carter, J.W. Jones, A.M. Farese, T.J. MacVittie, M.A. Kane, Inflation-fixation method for lipidomic mapping of lung biopsies by matrix assisted laser desorption/ionization-mass spectrometry imaging, Anal. Chem. 88 (2016) 4788-4794.

[12] C.L. Carter, C.W. McLeod, J. Bunch, Imaging of phospholipids in formalin fixed rat brain sections by matrix assisted laser desorption/ionization mass spectrometry, J. Am. Soc. Mass Spectrom. 22 (2011) 1991-1998.

[13] M. Pietrowska, M. Gawin, J. Polanska, P. Widlak, Tissue fixed with formalin and processed without paraffin embedding is suitable for imaging of both peptides and lipids by MALDI-IMS, Proteomics 16 (2016) 1670-1677.

[14] R.D. Addie, B. Balluff, J.V. Bovee, H. Morreau, L.A. McDonnell, Current state and future challenges of mass spectrometry imaging for clinical research, Anal. Chem. 87 (2015) 6426-6433.

[15] P.M. Vaysse, R.M.A. Heeren, T. Porta, B. Balluff, Mass spectrometry imaging for clinical research - latest developments, applications, and current limitations, Analyst 142 (2017) 2690-2712.

[16] N. Ogrinc Potocnik, T. Porta, M. Becker, R.M.A. Heeren, S.R. Ellis, Use of advantageous, volatile matrices enabled by next-generation high-speed matrixassisted laser desorption/ionization time-of-flight imaging employing a scanning laser beam, Rapid Commun. Mass Spectrom. 29 (2015) 2195-2203. 
[17] M.E. Belov, S.R. Ellis, M. Dilillo, M.R.L. Paine, W.F. Danielson, G.A. Anderson, E.L. de Graaf, G.B. Eijkel, R.M.A. Heeren, L.A. McDonnell, Design and performance of a novel interface for combined matrix-assisted laser desorption ionization at elevated pressure and electrospray ionization with Orbitrap mass spectrometry, Anal. Chem. 89 (2017) 7493-7501.

[18] S.R. Ellis, M.R.L. Paine, G.B. Eijkel, J.K. Pauling, P. Husen, M.W. Jervelund, M. Hermansson, C.S. Ejsing, R.M.A. Heeren, Automated, parallel mass spectrometry imaging and structural identification of lipids, Nat. Methods 15 (2018) 515-518.

[19] A. Palmer, P. Phapale, I. Chernyavsky, R. Lavigne, D. Fay, A. Tarasov, V. Kovalev, J. Fuchser, S. Nikolenko, C. Pineau, M. Becker, T. Alexandrov, FDR-controlled metabolite annotation for high-resolution imaging mass spectrometry, Nat. Methods 14 (2016) 57.

[20] M. Strohalm, M. Hassman, B. Kosata, M. Kodicek, mMass data miner: an open source alternative for mass spectrometric data analysis, Rapid Commun. Mass Spectrom. 22 (2008) 905-908.

[21] L. He, J. Diedrich, Y.Y. Chu, J.R. Yates 3rd, Extracting accurate precursor information for tandem mass spectra by RawConverter, Anal. Chem. 87 (2015) $11361-11367$.

[22] J.K. Pauling, M. Hermansson, J. Hartler, K. Christiansen, S.F. Gallego, B. Peng R. Ahrends, C.S. Ejsing, Proposal for a common nomenclature for fragment ions in mass spectra of lipids, PLoS One 12 (2017) e0188394.

[23] F.F. Hsu, J. Turk, Studies on sulfatides by quadrupole ion-trap mass spectrometry with electrospray ionization: structural characterization and the fragmentation processes that include an unusual internal galactose residue loss and the classical charge-remote fragmentation, J. Am. Soc. Mass Spectrom. 15 (2004) 536-546.

[24] R. Almeida, J.K. Pauling, E. Sokol, H.K. Hannibal-Bach, C.S. Ejsing, Comprehensive lipidome analysis by shotgun lipidomics on a hybrid quadrupoleorbitrap-linear ion trap mass spectrometer, J. Am. Soc. Mass Spectrom. 26 (2015) 133-148.

[25] P. Husen, K. Tarasov, M. Katafiasz, E. Sokol, J. Vogt, J. Baumgart, R. Nitsch, K. Ekroos, C.S. Ejsing, Analysis of lipid experiments (ALEX): a software framework for analysis of high-resolution shotgun lipidomics data, PLoS One 8 (2013) e79736.

[26] S. Vens-Cappell, I.U. Kouzel, H. Kettling, J. Soltwisch, A. Bauwens, S. Porubsky, J. Müthing, K. Dreisewerd, On-tissue phospholipase C digestion for enhanced MALDI-MS imaging of neutral glycosphingolipids, Anal. Chem. 88 (2016) 5595-5599.

[27] https://metaspace2020.eu/project/124f17f0-a498-11e9-9d77-17a7f6529a5a.

[28] H.Y. Wang, C.B. Liu, H.W. Wu, A simple desalting method for direct MALDI mass spectrometry profiling of tissue lipids, J. Lipid Res. 52 (2011) 840-849.

[29] P.M. Angel, J.M. Spraggins, H.S. Baldwin, R. Caprioli, Enhanced sensitivity for high spatial resolution lipid analysis by negative ion mode matrix assisted laser desorption ionization imaging mass spectrometry, Anal. Chem. 84 (2012) 1557-1564.

[30] X. Han, R.W. Gross, Structural determination of picomole amounts of phospholipids via electrospray ionization tandem mass spectrometry, J. Am. Soc. Mass Spectrom. 6 (1995) 1202-1210. 